\title{
A Dynamical System with Integer Information Dimension and Fractal Correlation Exponent*
}

\author{
C. D. Cutler
}

Department of Statistics and Actuarial Science,

University of Waterloo, Waterloo, Ontario, Canada N2L 3G1

\begin{abstract}
In this paper we construct a family $\left\{T_{\gamma}\right\}, 0<\gamma<\frac{1}{2}$, of exact endomorphisms of $[0,1]$ such that the invariant measure $m_{\gamma}$ of $T_{\gamma}$ is equivalent to Lebesgue measure but has fractal correlation exponent $v=2 \gamma$. This shows that an almost complete dichotomy can exist between the information dimension and the correlation exponent in observable dynamical systems.
\end{abstract}

\section{Introduction}

In the study of dynamical systems possessing an observable attractor $A$ and ergodic occupation measure $m$ two basic notions of dimension have developed. One is that of the fractal dimension of the attractor itself viewed as a geometrical object in space. The second is a measure-dependent notion which incorporates the actual dynamics of the system by taking into account the relative frequency with which a typical orbit visits different regions of the attractor. However even if we confine ourselves to one of these basic approaches the definition of dimension is not unique (see Farmer, Ott, and Yorke [3] and Hentschel and Procaccia [6]) although the various possible definitions may coincide in special cases. The fractal dimension $D$ of the attractor $A$ may be taken to be the Hausdorff dimension of $A$ or perhaps the capacity of $A$. Among measure-dependent notions the information dimension $\sigma$ and the correlation exponent $v$ (sometimes also called correlation dimension) have been widely discussed. Grassberger and Procaccia [4] introduced the correlation exponent as a measure-dependent gauge of dimension possessing the attractive feature of being easy to compute numerically from time series data. It was recognized immediately (Grassberger and Procaccia $[4,5]$ and Hentschel and Procaccia [6]) that for a large class of fractal attractors the general inequalities $v \leq \sigma \leq D$ hold with simultaneous equality $v=\sigma=D$ occurring if and only if the distribution is uniform across the attractor. However, in the estimation of dimension of a dynamical system from time series data, it appears to be a commonly held view that $v$ and $\sigma$ are generally close although not identical, and

\footnotetext{
* Research supported by the Natural Sciences and Engineering Research Council of Canada
} 
that observance of a small fractal correlation exponent is evidence of a small fractal information dimension and perhaps a strange attractor. (Related to this view is the idea that in systems governed by truly stochastic processes we should observe failure of the correlation exponents to converge during successive embeddings in higher-dimensional space. Hence observation of a finite value for $v$ is interpreted as evidence of low-dimensional chaos. Osborne and Provenzale [11] have discussed a counterexample to this supposition.)

In this paper we construct a simple piecewise monotonic transformation $T_{\gamma}$ (with parameter $\gamma, 0<\gamma<\frac{1}{2}$ ) on the unit interval having the properties:

$T_{\gamma}$ has an invariant distribution $m_{\gamma}$ which is equivalent to Lebesgue

measure and is also exact (hence mixing and ergodic).

$m_{\gamma}$ has correlation exponent $v=2 \gamma$.

The significance of (1.1) is twofold. From ergodicity and the equivalence of $m_{\gamma}$ to Lebesgue measure we see that $m_{\gamma}$ is obtained as the limiting occupation measure under repeated iterations of $T_{\gamma}$ for almost all initial conditions in $[0,1]$. (In fact in computer simulations the density of $m_{\gamma}$ appears clearly within a few thousand iterations.) Hence this is behavior which is actually observable rather than atypical conduct confined to sets of Lebesgue measure zero. Secondly the equivalence of $m_{\gamma}$ to Lebesgue measure implies that the attractor of the system is $[0,1]$ and that the information dimension of $m_{\gamma}$ equals 1 . Coupled with (1.2) this produces the result $v=2 \gamma<\sigma=D=1$. Since $\gamma$ can be chosen as close to zero as desired this shows that an almost complete dichotomy can exist between the correlation exponent and the information dimension in observable dynamical systems. This behavior is a consequence of the correlation exponent being more sensitive than the information dimension to nonuniformity in the distribution of points over the attractor. (In [2] Cutler has established an inequality between the correlation exponent and mean information dimension which holds under very general conditions.)

Precise definitions of all quantities are given in the next section.

\section{Definitions, the Transformation $T_{\gamma}$, and Statement of Theorem}

Let $m$ be a probability measure on the Borel sets of a compact subset $K$ of $\mathbb{R}^{N}$. The associated spatial correlation integral $C(r)$ is defined to be

$$
\begin{gathered}
C(r)=\iint I_{A_{r}}(x, y) m(d x) m(d y) \quad\left[\text { where } A_{r}=\{(x, y) \mid\|x-y\|<r\}\right] \\
=E(m(B(X, r))) \text { the mean or expected mass } \\
\text { in a random ball of radius } r .
\end{gathered}
$$

The correlation exponent $v$ is defined to be

$$
v=\lim _{r \rightarrow 0} \frac{\log C(r)}{\log r},
$$

provided the limit in (2.2) exists. There are various ways of defining the information dimension $\sigma$. These definitions coincide when $m$ is ergodic with respect to a 
sufficiently smooth transformation, so we will choose the simplest of these equivalent expressions

$$
\sigma=\inf \{\operatorname{dim}(B) \mid m(B)=1\},
$$

where $\operatorname{dim}(B)$ is the Hausdorff dimension of $B$. It is clear that $\sigma=1$ for any probability measure $m$ on $\mathbb{R}$ which is absolutely continuous with respect to Lebesgue measure.

We define the transformation $T_{\gamma}:[0,1] \rightarrow[0,1]$ for each $0<\gamma<\frac{1}{2}$ by

$$
T_{\gamma}(x)=\left\{\begin{array}{ll}
2 x & 0 \leq x<\frac{1}{2} \\
(2 x-1)^{1 / \gamma} & \frac{1}{2} \leq x \leq 1
\end{array} .\right.
$$

Note that $T_{\gamma}$ maps each of $\left(0, \frac{1}{2}\right)$ and $\left(\frac{1}{2}, 1\right)$ onto $(0,1)$ in a monotonic fashion and, while expanding on $\left(0, \frac{1}{2}\right)$, is contracting on a subinterval having $\frac{1}{2}$ as its lefthand endpoint. The result is that under iteration points are repeatedly pushed close to 0 and then repelled away and across the unit interval. The parameter $\gamma$ governs the degree of contraction and hence also the proportion of time interates spend near 0 . Various authors (see for example Hofbauer and Keller [7], Collet and Eckmann [1], Nowicki [10]) have investigated conditions under which maps of the interval will admit an ergodic invariant measure which is absolutely continuous with respect to Lebesgue measure. In this paper we rely on results of Misiurewicz [9] concerning piecewise monotone mappings as well as specific properties of $T_{\gamma}$ to show the existence of a measure $m_{\gamma}$ satisfying both (1.1) and (1.2). The calculations performed to determine bounds on the attracting density $f(x)$ [in order to establish (1.2)] simultaneously reveal the existence of at least one invariant measure $m_{\gamma}$ which is equivalent to Lebesgue measure. The existence theorem of Misiurewicz then assures us that $m_{\gamma}$ is exact and hence unique. Recall that an $m$-preserving mapping $T$ of $[0,1]$ onto itself is called an exact endomorphism if the tail $\sigma$-algebra $\mathscr{S}=\bigcap_{n \geq 1} T^{-n}(\mathfrak{B})$ (where $\mathfrak{B}$ is the $\sigma$-algebra of Borel sets) consists entirely of trivial events. That is, $m(A)=0$ or 1 for every $A \in \mathscr{S}$. It is well known that exact endomorphisms are mixing.

Remark. It can be shown (see the argument in the proof of Lemma 3.7) that the measure $m$ with density function $g(x)=\gamma x^{\gamma-1}$ has correlation exponent $v=2 \gamma$. In constructing $T_{y}$ the aim was to produce an attracting density $f(x)$ similar in behavior to $g(x)$. Of course $g(x)$ is noteworthy in itself since a sequence of independent and identically-distributed observations from this distribution provides an example of a completely random process on $[0,1]$ with fractal correlation exponent.

The conditions and results presented in Misiurewicz [9] are intended to cover a fairly general class of mappings and hence are more complicated than necessary for our simple map $T_{\gamma}$. Therefore we state a simplified special case of Theorems 6.2 and 6.3 of Misiurewicz [9] which will be directly applicable to $T_{\gamma}$.

Theorem 2.1 (Misiurewicz). Let $T:[0,1] \rightarrow[0,1]$ be a mapping for which there exists a point $c, 0<c<1$, such that $T$ is continuous and strictly monotone over each of the components $[0, c)$ and $(c, 1]$. Suppose further that $T(\{0,1\}) \subseteq\{0,1\}$, and both $\lim _{x \rightarrow c^{-}} T(x) \in\{0,1\}$ and $\lim _{x \rightarrow c^{+}} T(x) \in\{0,1\}$. Let $T$ also satisfy the following conditions: 
(a) $T$ belongs to the class $C^{3}$ on each of $(0, c)$ and $(c, 1)$.

(b) $D T(x) \neq 0$ for any $x \in(0,1) \backslash\{c\}$, where $D T$ denotes the derivative of $T$.

(c) $S T(x) \leq 0$ for all $x \in(0,1) \backslash\{c\}$, where $S T$ denotes the Schwarzian derivative $S T(x)=\frac{D^{3} T(x)}{D T(x)}-\frac{3}{2}\left(\frac{D^{2} T(x)}{D T(x)}\right)^{2}$.

This condition is equivalent to specifying that $\frac{1}{\sqrt{|D T(x)|}}$ is convex on each of $(0, c)$ and $(c, 1)$.

(d) If $x \neq c$ is a periodic point of period d then $\left|D T^{d}(x)\right|>1$.

(e) There exist positive real constants $\alpha_{0}, \omega_{0}, \alpha_{c^{-}}, \omega_{c^{-}}$, and $u_{0} \geq 0, u_{c^{-}} \geq 0$ such that, for some $\delta>0, \alpha_{0} x^{u_{0}} \leq|D T(x)| \leqq \omega_{0} x^{u_{0}}$ for $x \in(0, \delta)$ and $\alpha_{c^{-}}|x-c|^{u_{c}} \leqq|D T(x)|$ $\leqq \omega_{c^{-}}|x-c|^{u_{c}-}$ for $x \in(c-\delta, c)$. Similarly there exist positive real $\alpha_{1}, \omega_{1}, \alpha_{c^{+}}, \omega_{c^{+}}$, and $u_{1} \geq 0, u_{c^{+}} \geq 0$ such that, for some $\delta>0, \alpha_{1}|x-1|^{u_{1}} \leqq|D T(x)| \leqq \omega_{1}|x-1|^{u_{1}}$ for $x \in(1-\delta, 1)$ and $\alpha_{c^{+}}|x-c|^{u_{c}{ }^{+}} \leqq|D T(x)| \leqq \omega_{c^{+}}|x-c|^{u_{c}{ }^{+}}$for $x \in(c, c+\delta)$.

Then there exists a unique probability measure $m$ which is both T-invariant and absolutely continuous with respect to Lebesgue measure. Furthermore the system $([0,1], T, m)$ is exact.

The results concerning the behavior of $T_{\gamma}$ which we will establish are stated in the following theorem. $\lambda$ always denotes Lebesgue measure on $[0,1]$.

Theorem 2.2. Let $T_{\gamma}$ be defined as in (2.4). Then

(a) there exists a probability measure $m_{\gamma}$ on the Borel sets of $[0,1]$ which is invariant with respect to $T_{\gamma}$ (i.e. $m_{\gamma}=m_{\gamma} T_{\gamma}^{-1}$ ) and is equivalent to $\lambda$. Furthermore the density function $f(x)$ of $m_{\gamma}$ satisfies $C x^{\gamma-1} \leq f(x) \leq K x^{\gamma-1}$ for finite positive constants $C$ and $K$ (which depend on $\gamma$ ).

(b) The system $\left([0,1], T_{\gamma}, m_{\gamma}\right)$ is exact. Hence $T_{\gamma}$ is mixing and for each Borel set $B$ we have $\lim _{n \rightarrow \infty} \lambda T_{\gamma}^{-n}(B)=m_{\gamma}(B)$, and for $\lambda$-almost all $x \in[0,1]$ the sequence of empirical measures $\frac{1}{n} \sum_{j=1}^{n} \delta_{T^{j}(x)}$ converges weakly to $m_{\gamma^{*}}\left(\delta_{T^{j}(x)}\right.$ denotes the point mass
at $T^{j}(x)$.) (c) $m_{\gamma}$ has correlation exponent $v=2 \gamma$.

\section{Proof of Theorem 2.2}

The proof proceeds by a sequence of lemmas. We use the following notation: $m_{n}=\lambda T_{\gamma}^{-n}$ and $f_{n}(x)$ is the density function of $m_{n}$.

Note that the two solutions of $x=T_{\gamma}(z)$ are given by $z=\frac{x}{2}$ and $z=\frac{1+x^{\gamma}}{2}$. Hence the densities of $m_{n}$ and $m_{n+1}$ are related by the recursion formula (or PerronFrobenius operator):

$$
f_{n+1}(x)=\frac{1}{2} f_{n}\left(\frac{x}{2}\right)+\frac{\gamma}{2} x^{\gamma-1} f_{n}\left(\frac{1+x^{\gamma}}{2}\right)
$$

Lemma 3.1. For each $n \geq 1 f_{n}$ is a strictly decreasing function and $\sup _{n \geq 0} f_{n}\left(\frac{1}{2}\right) \leq 2$. 
Proof. This follows by induction on $n$. Note $f_{0}(x) \equiv 1$ is nonincreasing. Now if $f_{n}$ is strictly positive and nonincreasing then relation (3.1) shows that $f_{n+1}$ is strictly positive and strictly decreasing (since the functions $\frac{x}{2}, \frac{1+x^{\gamma}}{2}$, and $x^{\gamma-1}$ are respectively increasing, increasing, and strictly decreasing). This proves the first part of the lemma. The second part now follows from the inequalities

$$
\frac{1}{2} f_{n}\left(\frac{1}{2}\right) \leq \int_{0}^{1 / 2} f_{n}(x) d x \leq 1
$$

Lemma 3.2. Let $K=\frac{\gamma 2^{\gamma}}{2^{\gamma}-1}$. Then $\sup _{n \geq 0} f_{n}(x) \leq K x^{\gamma-1}$.

Proof. It is straightforward to show that $K \geq 1$, and hence $1 \equiv f_{0}(x) \leq K x^{\gamma-1}$. Now proceed by induction. Assume the lemma holds for $f_{n}$. By this assumption, Lemma 3.1, and relation (3.1) we obtain

$$
\begin{aligned}
f_{n+1}(x) & \leq \frac{1}{2} K\left(\frac{x}{2}\right)^{\gamma-1}+\frac{\gamma}{2} x^{\gamma-1} f_{n}\left(\frac{1}{2}\right) \\
& \leq \frac{1}{2} K\left(\frac{x}{2}\right)^{\gamma-1}+\frac{\gamma}{2} x^{\gamma-1}(2) \\
& =\left(\frac{K}{2^{\gamma}}+\gamma\right) x^{\gamma-1} \\
& =K x^{\gamma-1} \text { since } K=\frac{K}{2^{\gamma}}+\gamma .
\end{aligned}
$$

Lemma 3.3. There exist constants $C>0$ and $k \geq 1$ (depending on $\gamma$ ) such that $\inf _{n>k} f_{n}(x) \geq C x^{\gamma-1}$.

Proof. We first show there exist $k \geq 1$ and $a>0$ such that $f_{n}(x) \geq a$ for all $n \geq k$. From Lemma 3.2 we have $\int_{0}^{2-j} f_{n}(x) d x \leqq \int_{0}^{2-j} K x^{\gamma-1} d x=\frac{K}{\gamma} 2^{-j \gamma}$ for all $n$. Hence there exists $k \geq 1$ such that $\sup _{n \geq 1} \int_{0}^{2-k} f_{n}(x) d x \leqq \frac{K}{\gamma} 2^{-k \gamma}<\frac{1}{2}$, and therefore $\inf _{n \geq 1} \int_{2^{-k}}^{1} f_{n}(x) d x>\frac{1}{2}$. But from Lemma3.1 we also have $\int_{2^{-k}}^{1} f_{n}(x) d x$ $\leqq f_{n}\left(2^{-k}\right)\left(1-2^{-k}\right) \leqq f_{n}\left(2^{-k}\right)$ for each $n$, which shows that $\inf _{n \geq 0} f_{n}\left(2^{-k}\right) \geq \frac{1}{2}$. Now from relation (3.1) and nonnegativity we see that $f_{n}(x) \geq \frac{1}{2} f_{n-1}\left(\frac{x}{2}\right)$ and hence, inductively, for all $n \geq k$ we conclude $f_{n}(1) \geq 2^{-k} f_{n-k}\left(2^{-k}\right) \geqq 2^{-k} 2^{-1}=2^{-(k+1)}$. Since $f_{n}$ is decreasing this shows $\inf _{n \geq k} f_{n}(x) \geq a$, where $a=2^{-(k+1)}$. Now using relation (3.1) with $n \geq k$ we obtain $f_{n+1}(x) \geq \frac{\gamma}{2} x^{\gamma-1} f_{n}\left(\frac{1+x^{\gamma}}{2}\right) \geq \frac{a \gamma}{2} x^{\gamma-1}$. Therefore the lemma is proved with $C=\frac{a \gamma}{2}$. 
Lemma 3.4. Let $\bar{m}_{n}=\frac{1}{n} \sum_{j=1}^{n} m_{j}$. Then $\left\{\bar{m}_{n}\right\}_{n}$ has a weakly convergent subsequence. The limit $m_{\gamma}$ of this subsequence is equivalent to Lebesgue measure and has density function $f$ such that $C x^{\gamma-1} \leq f(x) \leq K x^{\gamma-1}$. Moreover $m_{\gamma}$ is invariant with respect to $T_{\gamma}$.

Proof. As is well known the existence of a weakly convergent subsequence with limit $m_{\gamma}$ follows from the compactness of $[0,1]$. The invariance of $m_{\gamma}$ is also immediate provided the set of discontinuities $\mathscr{D}$ of $T_{\gamma}$ has $m_{\gamma}$-measure 0 . Since $\mathscr{D}=\left\{\frac{1}{2}\right\}$ this will certainly be true if $m_{\gamma}$ is equivalent to Lebesgue measure. Thus it remains only to prove this last assertion with the stated bounds on the density $f$. But from Lemma 3.3 we see that, for all sufficiently large $n, m_{\ell} \leq m_{n} \leq m_{u}$, where $m_{\ell}$ and $m_{u}$ are the measures with densities $f_{\ell}(x)=C x^{\gamma-1}$ and $f_{u}(x)=K x^{\gamma-1}$ respectively. It follows that for each Borel set $B$,

$$
m_{\ell}(B) \leq \liminf _{n \rightarrow \infty} \bar{m}_{n}(B) \leq \limsup _{n \rightarrow \infty} \bar{m}_{n}(B) \leqq m_{u}(B)
$$

Hence we must have $m_{\ell} \leq m_{\gamma} \leq m_{u}$ and the lemma follows.

Lemmas 3.1-3.4 prove (a) of Theorem 2.2. We need the following lemma to show (b) of Theorem 2.2. Let $\mathscr{Z}=\bigcup_{n=0}^{\infty} T_{\gamma}^{-n}(0)$.

Lemma 3.5. If $x \in(0,1) \backslash \mathscr{Z}$, then $\limsup _{n \rightarrow \infty} D T_{\gamma}^{n}(x)=\infty$.

Proof. By the chain rule $D T_{\gamma}^{n}(x)=\prod_{j=0}^{n-1} D T_{\gamma}\left(T_{\gamma}^{j}(x)\right)$. Now if $0<u<\frac{1}{2}$, we have $D T_{\gamma}(u)=2$ while if $\frac{1}{2}<u<1$ we have $D T_{\gamma}(u)=\frac{2}{\gamma}(2 u-1)^{\frac{1}{\gamma}}-1$. Write $\left(\frac{1}{2}, 1\right)=I \cup J$, where $I=\left(\frac{1}{2}, \frac{1+2^{-\gamma}}{2}\right)$ and $J=\left(\frac{1+2^{-\gamma}}{2}, 1\right)$. Then $u \in J$ implies $D T_{\gamma}(u) \geq \frac{1}{\gamma}>2$. We observe that $D T_{\gamma}(u)$ is small only for $u \in I$. So suppose $u \in I$. Then there exists $n \geq 2$ such that $a_{n} \leq u<a_{n-1}$, where $a_{j}=\frac{1+2^{-j \gamma}}{2}$. Hence

$$
D T_{\gamma}(u) \geq D T_{\gamma}\left(a_{n}\right)=\frac{2}{\gamma}\left(2 a_{n}-1\right)^{\frac{1}{\gamma}-1}=\gamma^{-1} 2^{1-n(1-\gamma)} .
$$

Now $T_{\gamma}(u)<T_{\gamma}\left(a_{n-1}\right)=\left(2 a_{n-1}-1\right)^{1 / \gamma} \leq 2^{-(n-1)}$, and so it follows that $D T_{\gamma}\left(T_{\gamma}^{j}(u)\right)=2$ for $j=1,2, \ldots, n-1$. Consequently we have

$$
D T_{\gamma}^{n}(u)=\prod_{j=0}^{n-1} D T_{\gamma}\left(T_{\gamma}^{j}(u)\right) \geq \gamma^{-1} 2^{1-n(1-\gamma)} 2^{n-1}>\frac{1}{\gamma}>2 .
$$

From this it is clear that for any $x \in(0,1) \backslash \mathscr{Z}$ there exists a subsequence $n_{k}$ such that $\lim _{k \rightarrow \infty} D T_{\gamma}^{n_{k}}(x)=\infty$.

Lemma 3.6. $T_{\gamma}$ satisfies conditions (a)-(e) of Theorem 2.1 . 
Proof. Conditions (a), (b), (c) are trivial to verify. To see (e) note that $D T_{\gamma}(x)=2$ on $\left(0, \frac{1}{2}\right), D T_{\gamma}(x)=\alpha\left(x-\frac{1}{2}\right)^{u}$ on $\left(\frac{1}{2}, 1\right)$, where $u=\gamma^{-1}-1$ and $\alpha=2^{u+1} \gamma^{-1}$, and $1 \leq D T_{\gamma}(x) \leq 2 \gamma^{-1}$ for all $x$ sufficiently close to 1 . We now need only check (d). The only fixed points $x=0$ and $x=1$ are clearly repelling. Therefore consider interior points $x$ for which $T_{\gamma}^{d}(x)=x$, and note that $D T_{\gamma}^{k d}(x)=\left(D T_{\gamma}^{d}(x)\right)^{k}$. Hence the sequence $D T_{\gamma}^{k d}(x), k=1,2, \ldots$ is bounded if $D T_{\gamma}^{d}(x) \leq 1$. But from Lemma 3.5, the chain rule, and the fact that $\sup _{0<u<1} D T_{\gamma}(u)=2 \gamma^{-1}<\infty$, we see that the sequence $D T_{\gamma}^{k d}(x)$, $k=1,2, \ldots$ cannot be bounded. Hence (d) holds.

Thus Misiurewicz's conditions are verified and we conclude $T_{\gamma}$ is exact. The remaining claims in (b) of Theorem 2.2 are a consequence of mixing (see for example Proposition 8.3, p. 143, Mañé [8].)

Lemma 3.7. Let $m_{\gamma}$ be the distribution obtained in Theorem 2.2(a). Then there exist finite positive constants $A$ and $B$ (depending on $\gamma$ ) such that $A \leqq \liminf _{r \rightarrow 0} \frac{C(r)}{r^{2 \gamma}}$ $\leq \limsup _{r \rightarrow 0} \frac{C(r)}{r^{2 \gamma}} \leq B$. Consequently $v=2 \gamma$.

Proof. Since the density $f(x)$ of $m_{\gamma}$ satisfies the bounds $C x^{\gamma-1} \leq f(x) \leqq K x^{\gamma-1}$ it will be sufficient to consider the behavior of a measure $m$ with density $g(x)=M x^{\gamma-1}$ for some positive constant $M$. Define $G(x)=\int_{0}^{x} M t^{\gamma-1} d t=M^{*} x^{\gamma}$ $\left(\right.$ where $\left.M^{*}=\frac{M}{\gamma}\right)$ and note that we can write

$$
C(r)=E(m(B(X, r)))=I_{1}(r)+I_{2}(r)+I_{3}(r),
$$

where

$$
\begin{aligned}
& I_{1}(r)=\int_{0}^{r} G(x+r) g(x) d x \\
& I_{2}(r)=\int_{r}^{1-r}(G(x+r)-G(x-r)) g(x) d x,
\end{aligned}
$$

and

$$
I_{3}(r)=\int_{1-r}^{1}\left(M^{*}-G(x-r)\right) g(x) d x
$$

Now

$$
I_{3}(r) \leq \int_{1-r}^{1} M^{*} g(x) d x \leq M^{*} r g\left(\frac{1}{2}\right)
$$

for $0<r \leq \frac{1}{2}$. Hence we obtain

$$
\lim _{r \rightarrow 0} r^{-2 \gamma} I_{3}(r) \leq \lim _{r \rightarrow 0} M^{*} g\left(\frac{1}{2}\right) r^{1-2 \gamma}=0 .
$$


Now consider $I_{1}(r)$. Making the substitution $u=\frac{x}{r}$, we can write

$$
I_{1}(r)=M M^{*} \int_{0}^{r}(x+r)^{\gamma} x^{\gamma-1} d x=r^{2 \gamma} M M^{*} \int_{0}^{1}(1+u)^{\gamma} u^{\gamma-1} d u
$$

which gives $\lim _{r \rightarrow 0} r^{-2 \gamma} I_{1}(r)=M M^{*} I_{1}$, where $I_{1}=\int_{0}^{1}(1+u)^{\gamma} u^{\gamma-1} d u$. This leaves $I_{2}(r)$. This time make the substitution $u=\frac{r}{x}$ to obtain

$$
\begin{aligned}
I_{2}(r) & =M M^{*} \int_{r}^{1-r}\left((x+r)^{\gamma}-(x-r)^{\gamma}\right) x^{\gamma-1} d x \\
& =r^{2 \gamma} M M^{*} \int_{\frac{r}{1-r}}^{1}\left((1+u)^{\gamma}-(1-u)^{\gamma}\right) u^{-(1+2 \gamma)} d u .
\end{aligned}
$$

Hence $\lim _{r \rightarrow 0} r^{-2 \gamma} I_{2}(r)=M M^{*} I_{2}$, where $I_{2}=\int_{0}^{1}\left((1+u)^{\gamma}-(1-u)^{\gamma}\right) u^{-(1+2 \gamma)} d u$. (Note it is easy to see that $I_{2}$ is finite by applying the Mean Value Theorem to the function $x^{\gamma}$ evaluated at the endpoints $1-u$ and $1+u$.) We conclude $\lim _{r \rightarrow 0} r^{-2 \gamma} C(r)$ $=M M^{*}\left(I_{1}+I_{2}\right)$ for the measure $m$. From this we see that for the measure $m_{\gamma}$ the conclusion of the lemma must hold with $A=\gamma^{-1} C^{2}\left(I_{1}+I_{2}\right)$ and $B=\gamma^{-1} K^{2}\left(I_{1}+I_{2}\right)$.

This completes the proof of Theorem 2.2.

Concluding Remarks. This paper illustrates the need to exercise caution when drawing conclusions about the dimension structure of a dynamical system based on a single method of dimension analysis. It indicates that we need to have a clear understanding of the differences between the various definitions of dimension (and suggests also that perhaps the correlation exponent should not be regarded as a dimension at all). While this example may appear somewhat artificial because of its one-dimensional phase space and simple mechanism (which would be easily identified by any observer of the system) it would not be difficult to embed maps with similar properties into high-dimensional systems where any simple behavior would be obscured by the general complexity of the dynamics. Estimates of the correlation exponent from such a system would be considerably lower than the actual information dimension of the system. (Indeed we have seen that $v$ and $\sigma$ can be made as far apart as desired, up to the dimension of the phase space.) Real insight into the behavior of a dynamical system would appear to be obtained by studying jointly the three quantities $v, \sigma$, and $D$ rather than any one in isolation.

\section{References}

1. Collet, P., Eckmann, J.-P.: Positive Liapunov exponents and absolute continuity for maps of the interval. Ergod. Th. Dynam. Sys. 3, 13-46 (1983)

2. Cutler, C.D.: Some results on the behavior and estimation of the fractal dimensions of distributions on attractors, preprint (1989) 
3. Farmer, J.D., Ott, E., Yorke, J.A.: The dimension of chaotic attractors. Physica D 7, 153-180 (1983)

4. Grassberger, P., Procaccia, I.: Characterizations of strange attractors. Phys. Rev. Lett. 50, 346-349 (1983)

5. Grassberger, P., Procaccia, I.: Measuring the strangeness of strange attractors. Physica D 9, 189-208 (1983)

6. Hentschel, H.G.E., Procaccia, I.: The infinite number of generalized dimensions of fractals and strange attractors. Physica D 8, 435-444 (1983)

7. Hofbauer, F., Keller, G.: Ergodic properties of invariant measures for piecewise monotonic transformations. Math. Z. 180, 119-140 (1982)

8. Mañé, R.: Ergodic theory and differentiable dynamics. Berlin, Heidelberg, New York: Springer 1987

9. Misiurewicz, M.: Absolutely continuous measures for certain maps of an interval. Publ. Math. IHES 53, 17-51 (1981)

10. Nowicki, T.: Symmetric $S$-unimodal mappings and positive Liapunov exponents. Ergod. Th. Dynam. Sys. 5, 611-616 (1985)

11. Osborne, A.R., Provenzale, A.: Finite correlation dimension for stochastic systems with power-law spectra. Physica D 35, 357-381 (1989)

Communicated by J.-P. Eckmann

Received September 29, 1989; in revised form October 30, 1989 
